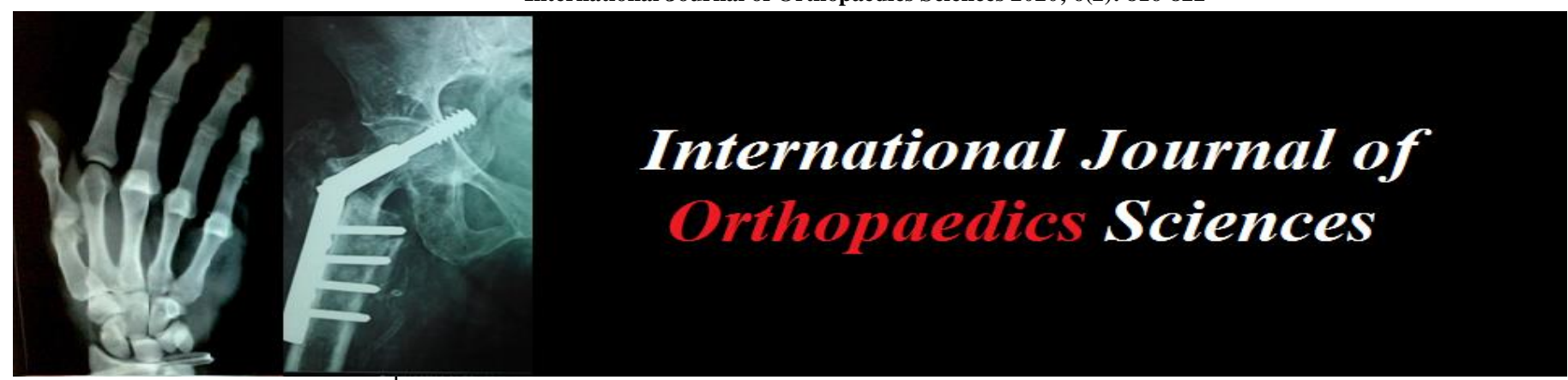

E-ISSN: 2395-1958

P-ISSN: 2706-6630

IJOS 2020; 6(2): 816-822

(C) 2020 IJOS

www.orthopaper.com

Received: 25-02-2020

Accepted: 27-03-2020

Dr. Venkataraman Manian

Associate Professor,

Orthopaedics, Chettinad

Hospital and Research Institute,

Kelambakkam, Tamil Nadu,

India

Dr. Victor Moirangthem

Professor, Department of

Orthopaedics, Chettinad

Hospital \& Research Institute,

Kelambakkam, Kanchipuram,

Tamil Nadu, India

Dr. Arun Kumar KV

Assistant Professor, Orthopedics Chettinad Academy of Research and Education, Kelambakkam,

Tamil Nadu, India

Dr. Alagesan Shanmugavel Consultant Orthopaedic Surgeon, Chettinad Hospital and Research Institute, Kelambakkam,

Tamil Nadu, India
Corresponding Author: Dr. Victor Moirangthem Professor, Department of Orthopaedics, Chettinad

Hospital \& Research Institute, Kelambakkam, Kanchipuram,

Tamil Nadu, India

\section{Outcome of total knee replacement in osteoarthritis: A prospective study}

\author{
Dr. Venkataraman Manian, Dr. Victor Moirangthem, Dr. Arun Kumar KV \\ and Dr. Alagesan Shanmugavel
}

DOI: https://doi.org/10.22271/ortho.2020.v6.i2m.2144

\section{Abstract}

Background: Severe osteoarthritis of the knee cause pain and disability that affect the livelihood of people especially in rural areas and reduce the quality of life in general. Total knee arthroplasty is a cost-effective treatment for the surgical management of advanced osteoarthritis of the knee when other treatment options become ineffective.

Objectives: To analyze the results of total knee arthroplasty for osteoarthritis of the knee using knee society score.

Materials and Methods: This was a prospective study. The duration of study period was 4 years. 40 patients with osteoarthritis of the knee satisfying the inclusion criteria and who had given consent for posterior stabilized total knee arthroplasty during the period of January 2015 to December 2018 were included in the study. The patients were followed up at one, three and six months and then every year. They were evaluated radiographically and functionally using the Knee Society Score at each visit.

Results: There was significant improvement in knee society scores as compared to the respective preoperative treatment values. Majority of the patients (34) have knee society score (KSS) above 80 at final follow-up. One of the patients had surgical site infection which settled down with wound debridement and intravenous antibiotics. His final KSS was poor at last follow up.

Conclusions: Total knee arthroplasty is an effective treatment for moderate to severe osteoarthritis of the knee in terms of improved quality of life.

Keywords: Primary total knee arthroplasty, osteoarthritis, knee society score

\section{Introduction}

Osteoarthritis (OA) of the knee is a ubiquitous disease among people above 65 years of age and involves about $3.5 \%$ of the people worldwide. It causes profound disability in advanced stage of the disease when the pain is associated with deformity, restriction of mobility and radiographic evidence of progressive joint cartilage loss ${ }^{[1,2]}$. Various studies have shown that $\mathrm{OA}$ of knee is more common in the females. Primary OA is the more commonly encountered type due to cartilage wear and tear whereas the secondary variety may result from joint injuries, inflammatory diseases or overweight ${ }^{[3,4]}$.

Majority of the patients with OA of the knee can be initially managed without surgery. Some of the non-operative treatments include patient education, aerobic and strengthening exercises, weight management, transcutaneous electrical nerve stimulation (TENS), medial patellar taping, braces, canes, pain management with NSAIDS, acetaminophen and opioids ${ }^{[2,4]}$. Intra-articular steroids, viscosupplementations and biologics have been shown in many studies to give shortterm benefits. Arthroscopic debridement and high tibial osteotomy have been used in selected cases with limited success ${ }^{[1]}$.

Total Knee Arthroplasty (TKA) remains the gold standard for the management of severe osteoarthritis of the knee (Kellgren and Lawrence Grade 3 and 4) as it has shown to be efficacious in term of elimination of pain and regain of knee function by several authors ${ }^{[5]}$. The techniques of TKA has evolved through the year from Fergusson's resection arthroplasty to robotic knee replacement ${ }^{[6]}$.

The purpose of this study is to analyze the result of posterior stabilized TKA for severe osteoarthritis using the Knee Society Score, which incorporated in a single system separate knee and patient function scores ${ }^{[7]}$. 


\section{Materials and Methods}

The study was conducted between January 2015 to December 2018 on forty (40) consecutive patients with severe osteoarthritis of the knee. Inclusion criteria were patients who have uni- or bilateral Kellgren and Lawrence grade 3 and 4 osteoarthritis of the knee with significant limitations of activities of daily living and have failed non-operative treatment. Localized or remote active infection, ipsilateral hip disease, spinal stenosis or radicular symptoms, neuromuscular diseases and unwilling patients are excluded from the study. Informed written consent were obtained from all study participants.

All study participants were interviewed in detail about their complaints and expectations from the treatment. Pre-operative knee society score was recorded for all the patients. A comprehensive biochemical and blood profiles studies as part of any major surgical procedures were ordered. Pre-operative assessment was routinely done by the medical and anaesthetic teams. Standard orthogonal and patellar radiographs were studied to determine the severity of joint disease, bony defects, osteophytes, insufficiency of supporting ligamentous structures, trochlear defect and these was supplemented with full-length standing anteroposterior radiograph including the hip, knee and ankle joints to calculate anatomic varus angle and medial tibial proximal angle.

The surgeries were performed under combined epidural and spinal anaethesia and a first-generation cephalosporin antibiotic (usually cefuroxime $1 \mathrm{gm}$ ) was given thirty minutes before the tourniquet was inflated. The same antibiotic was continued for 24 hours. The patella was not resurfaced in all our cases. With the prepped knee in 90 degrees of flexion, an anterior midline skin incision was made, followed sequentially by medial parapatellar arthrotomy, lateral retraction of the patella, deep medial collateral ligament release and osteophytes removed from both the femoral and tibial condyles. Distal femoral resection was done at 5 degrees valgus angles in all the knees and the proximal tibia resected perpendicular to its mechanical axis using an external jig, Femoral rotation was measured using the Whitesides line and the transepicondylar axis and the size determined using posterior referencing guide. The anterior femoral, posterior condylar and chamfer cuts were then completed using the 4-in-1 guide. Since we used posterior stabilized knee in all our cases, we completed the femoral box using the box cutting guide. Tibial keel was then prepared using the jig and component rotation set by aligning with the medial third of tibial tubercle. Patellar resurfacing was not performed in all the patients but was denervated circumferentially after removal of marginal osteophytes. Trial components were then inserted with $9 \mathrm{~mm}$ spacer and checked for the need of additional soft tissue release or malpositioned components or patellar maltracking. Finally, cementing of the components were completed in a single stage. Wounds were closed in layers over a negative suction drain in all the cases. We routinely instill $3 \mathrm{gms}$ of intra-articular tranexamic acid after wound closure for haemostasis.

In the postoperative period, we used epidural analgesia for first twenty-four hours, followed by NSAIDS and transdermal opioid patch. Drains were removed the following day and patients were made to bear weight on the first post-operative day. Walking and knee movements slowly commenced according to the patient comfort level. Deep vein thrombosis (DVT) prophylaxis was initiated 12 hours after surgery using low molecular weight heparin, continued till discharge and then substituted with aspirin $150 \mathrm{mg}$ tablets for fourteen days. Radiographs were ordered in the immediate postoperative day and on every visit. The patients were discharged on $5^{\text {th }}$ day after surgery. They were reviewed after a week, at one month, 3 months, 6 months, and every year thereafter. The KSS was recorded on each visit after completing six months following surgery.

\section{Results}

Out of the 40 patients included in the study, twenty-five $(62.5 \%)$ were females and fifteen $(37.5 \%)$ were males. There were five $(12.5 \%)$ bilateral knee arthroplasties, making a total of 45 knees. The number of right knee cases was 18(40\%) and those of the left knee was $27(60 \%)$ showing a left knee predominance. The mean age was 71.51 years (Range: 65 to 85 years). The blood loss in the perioperative period calculated as the difference between the preoperative and fifth postoperative day haemoglobin had a mean value of $2.84 \mathrm{gm} / \mathrm{dl}$ (Range: 2 to 4). Most of the patients were discharged from the hospital at an average of 5.42 days (Range: 5 to 7 ) after surgery. The mean duration of follow-up was 35.20 months (Range: 24 to 48).

The preoperative KSS Knee and patient function scores were poor which improved significantly after the procedure. Twenty-two (48.89\%) patients had an excellent outcome, sixteen $(35.55 \%)$ gave good result, six $(13.33 \%)$ had fair grade at the most recent follow-up. One $(2.22 \%)$ male patient with diabetes and moderate obesity developed delayed wound healing with persistent discharge who had to be taken to operation theatre for wound debridement. Coagulase negative staphylococcus was isolated from joint fluid and intravenous linezolid 600mg every 12 hourly was continued for three weeks then orally for another two weks which settled the infection. The patient had persistent mild residual pain, restriction of knee movement and his KSS remained poor. After twenty-four months of follow-up, x-rays showed no signs of lucencies around the components.

The following tables summarized the pre- and post-operative $\mathrm{KSS}$ and the respective tests for significance in the difference of their mean values.

Table 1: Paired Sample Statistics

\begin{tabular}{|c|c|c|c|c|c|}
\hline \multicolumn{2}{|c|}{ Paired Samples Statistics } & Mean & N & Std. Deviation & Std. Error Mean \\
\hline \multirow{2}{*}{ Pair 1 } & Postop KSS Function & 74.98 & 45 & 6.910 & 1.030 \\
\cline { 2 - 6 } & Preop KSS Function & 50.00 & 45 & 4.129 & .615 \\
\hline \multirow{2}{*}{ Pair 2 } & Postop KSS Clinical & 75.67 & 45 & 5.394 & .804 \\
\cline { 2 - 6 } & Preop KSS Clinical & 51.11 & 45 & 3.827 & .571 \\
\hline
\end{tabular}


Table Paired Samples Test

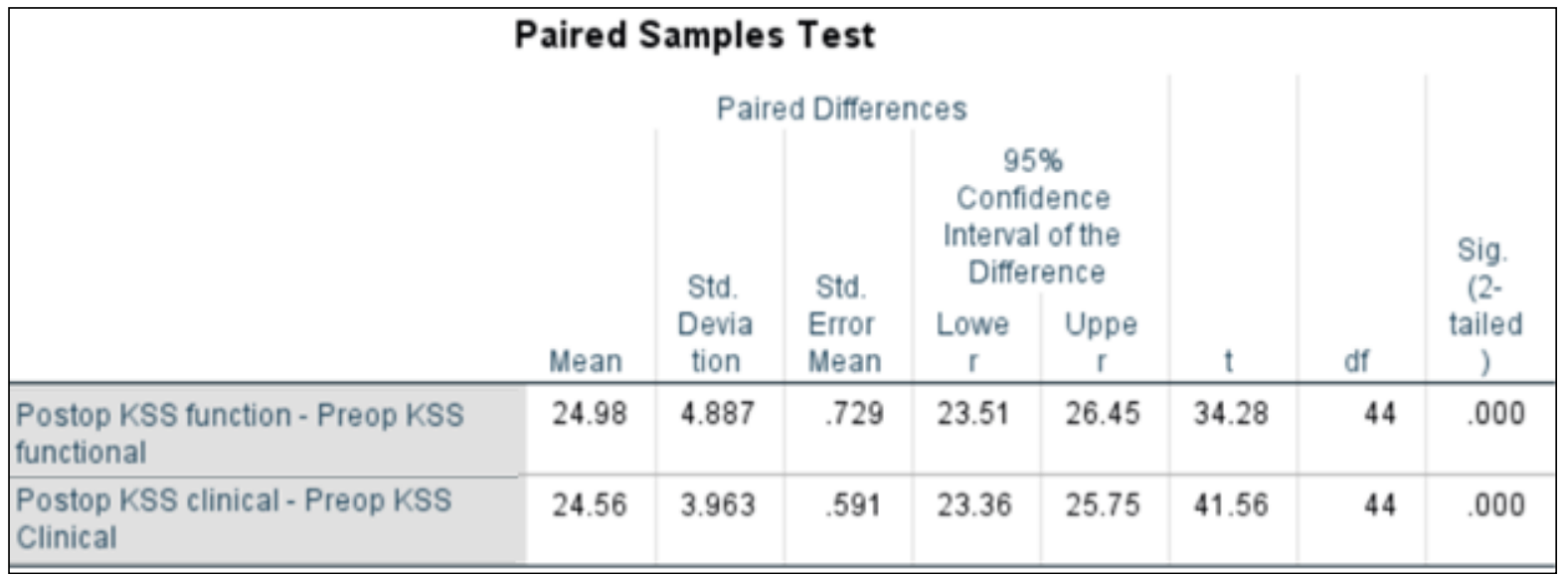

The mean preoperative KS functional score improved from $50.00 \pm 4.13$ to $74.98 \pm 6.91$ postoperatively. Similarly, the mean preoperative $\mathrm{KS}$ clinical/knee score which was $51.11 \pm 3.83$ increased to $75.67 \pm 5.39$ after surgery. The differences in these means were found to be statistically significant $(p<0.00)$.

Discussions

Although both posterior cruciate retaining (CR) and posterior stabilized (PS) knees have been demonstrated by various studies to give similar outcome, Lombardi proposed PS knees for elderly patients with severe arthritis with significant deformities in both planes. Most of our study participants belongs to the category for PS knees as described in Lombardi's algorithm ${ }^{[8]}$.

Scott in his study of 56 knees found the average age was 67.7 with predominantly female patients ${ }^{[9]}$. Wilton reported a higher mean age of 73.2 years and a mean follow-up of 3.5 years ${ }^{[10]}$. Similarly, the mean age of our patients were 71.51 years with an average follow-up of 2.9 years.

The usual practice of measuring perioperative blood loss from the amount of intra-operative and post-operative drainage fails to take into account the unaccounted loss into tissues, knee and haemolysis as mentioned by Sehat, who further emphasized upon the need to measure pre-operative and post-operative haematocrit to accurately calculate the blood loss ${ }^{[11]}$. Zi-Jian $\mathrm{Li}$ concluded that the Hb-balance method may be the most reliable method for estimating blood loss after TKA ${ }^{[12]}$. Lisander accepted that the blood volume on the fifth day after surgery was the same as that before surgery ${ }^{[13]}$. Measuring the difference in haemoglobin between the pre-operative and $5^{\text {th }}$ post-operative value therefore gives a reliable estimate of the amount of blood loss during TKA. Our mean loss of $2.84 \mathrm{gm} \%$ was comparable with values reported by Wong ${ }^{[14]}$.

Yang found in a meta-analysis that tranexamic acid given topically was equally efficacious as intravenous tranexamic acid in reducing both blood loss and transfusion rate without sacrificing safety in primary total knee arthroplasty ${ }^{[15]}$. Patel et al. reported a similar finding ${ }^{[16]}$.

A recent meta-analysis concluded that aspirin, both alone and in multimodal approaches to thromboprophylaxis, confers a low rate of VTE, with a low risk of major bleeding complications ${ }^{[17]}$

Meding showed in a study of 128 patients followed for a minimum of twenty years, that the KS knee/clinical scores moved from a mean value of $54 \pm 11$ before TKA to a mean value of $78 \pm 14$ following the procedure. For the same group, the KS functional scores improved from a preoperative mean value of $36 \pm 20$ to an average value of $70 \pm 24$ postoperatively.
He however emphasized that the decline in KS function value was due to diminished physical function from increase life expectancy and was independent of prosthetic function ${ }^{[18]}$. We were able to achieve a similar result in this study.

The mean preoperative mechanical alignment was $7.67 \pm 11.90$ of varus (range, $25^{\circ}$ of varus to $20^{\circ}$ of valgus) which were corrected after TKA to a mean value of $2.27 \pm 3.89$ of valgus (range, $7^{\circ}$ of varus to $12^{\circ}$ of valgus). One of patient had preoperative valgus of $20^{\circ}$ on both knees and a sequential bilateral TKA was performed. We were able to achieve up to 10 degrees of correction. We had used a cemented tibial component stem extension in view of her severe deformity. Kathryn K. Oi reported that the indication for stem use in primary TKA were the presence of implants, higher BMI, severe deformity, and it was more commonly used in female patients ${ }^{[19]}$.

Diabetes mellitus, poor nutrition, obesity, extreme old age, ancient septic arthritis, and prolonged hospitalization have been described as risk factors for infection after total knee arthroplasty ${ }^{[20]}$. A four to six weeks of intravenous antibiotics sensitive to the infecting organism was recommended for prosthetic joint infection ${ }^{[21]}$.

\section{Illustrative cases}

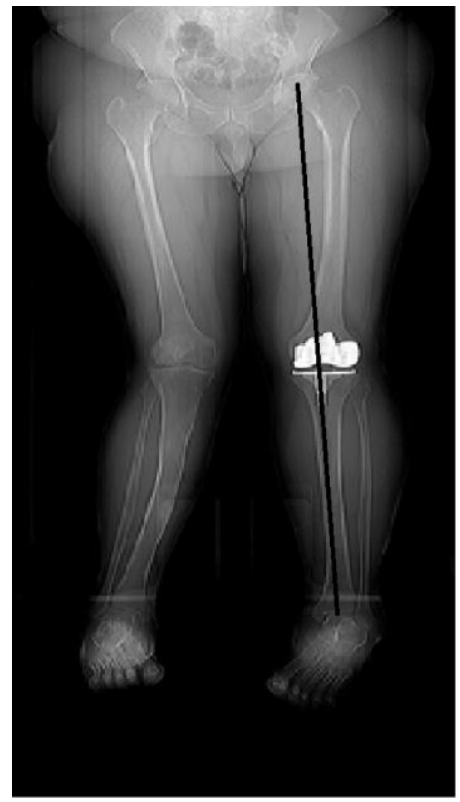

Post-operative CT Scanogram of a patient showing mechanical axis. 

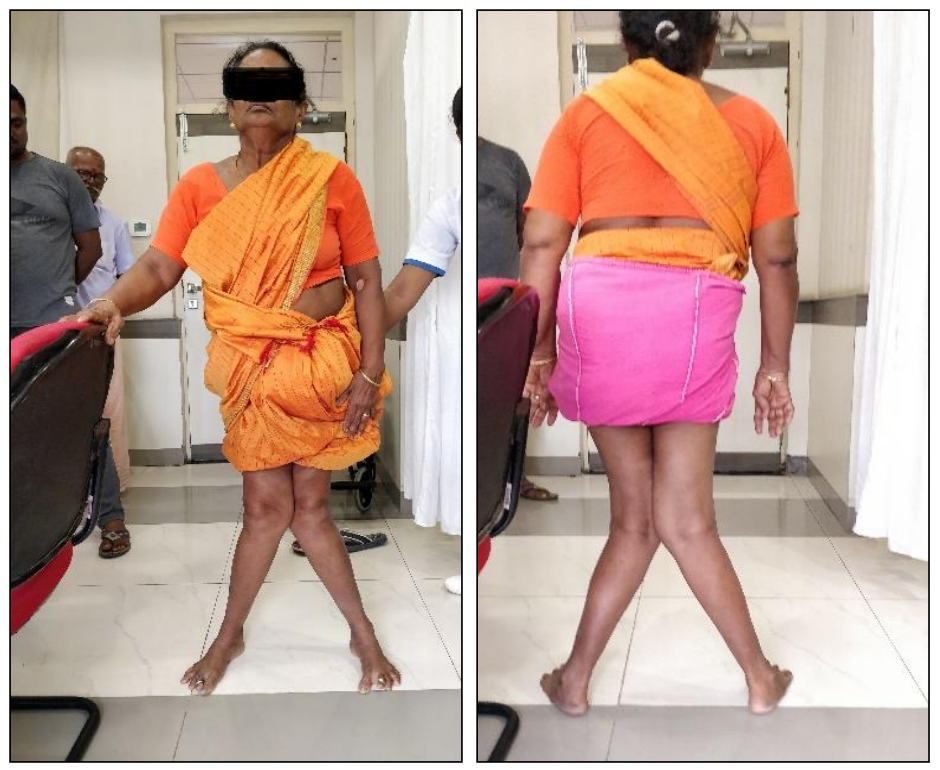

Pre-operative Clinical Image of a patient with severe valgus knees
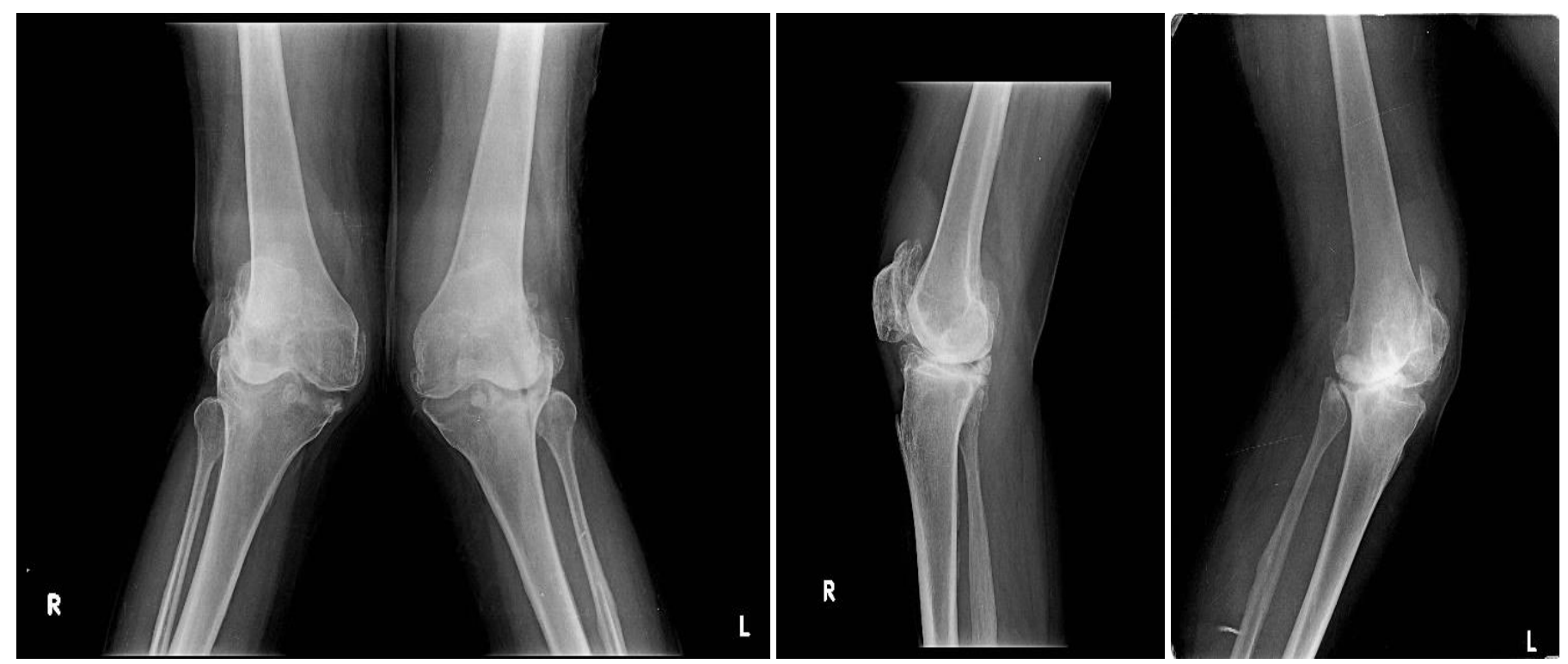

Antero-posterior and lateral pre-operative radiograph of above female patient

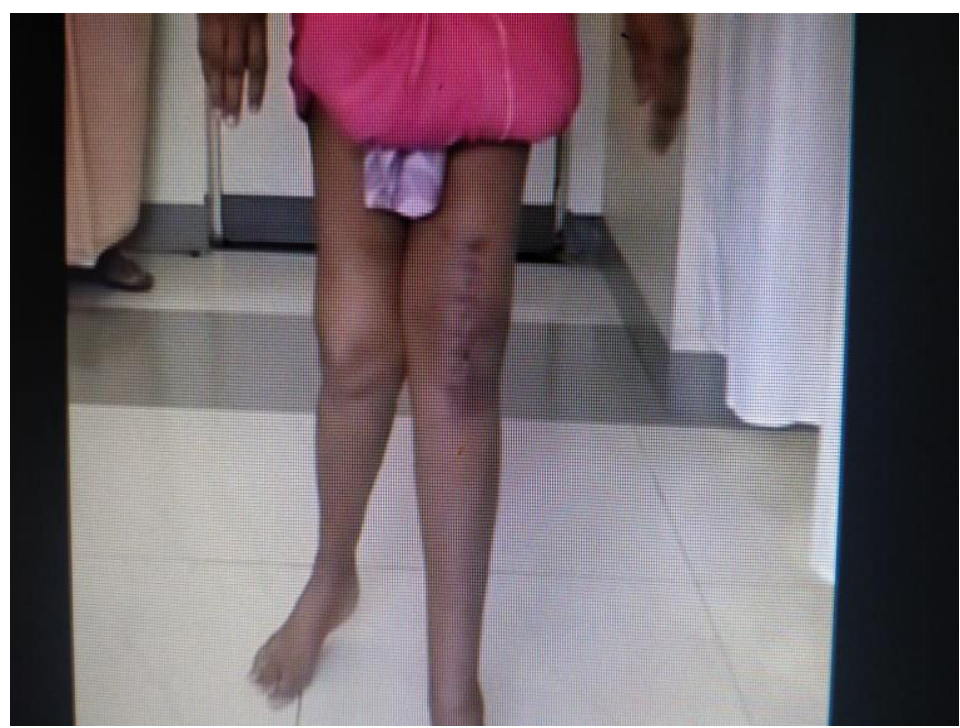

Same patient after TKA on the left knee 


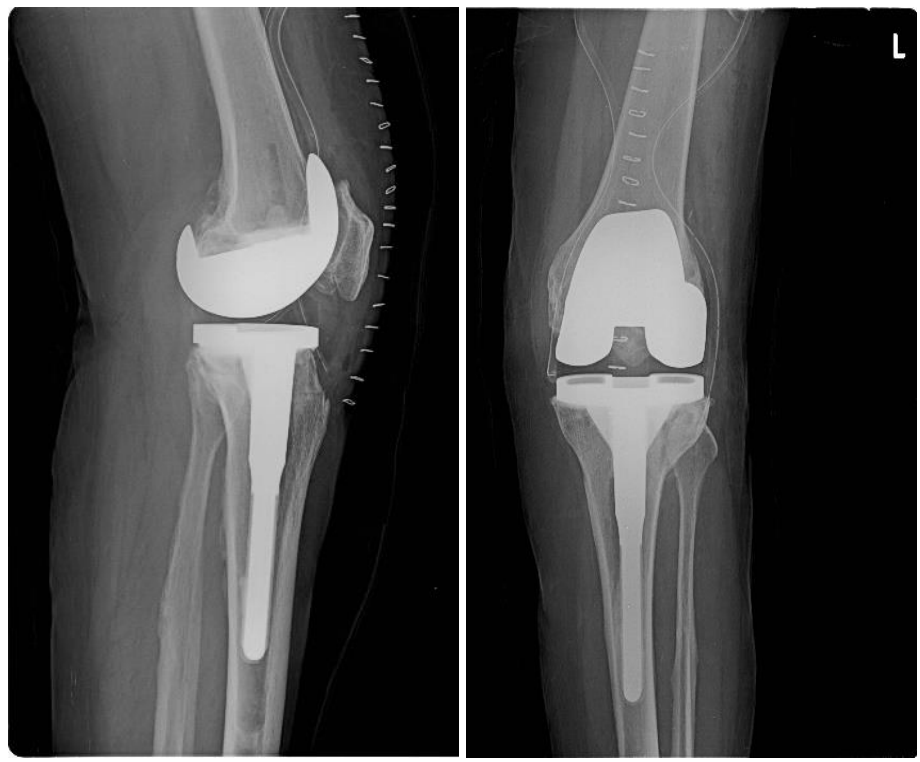

Post-operative radiograph showing reasonable correction of valgus deformity

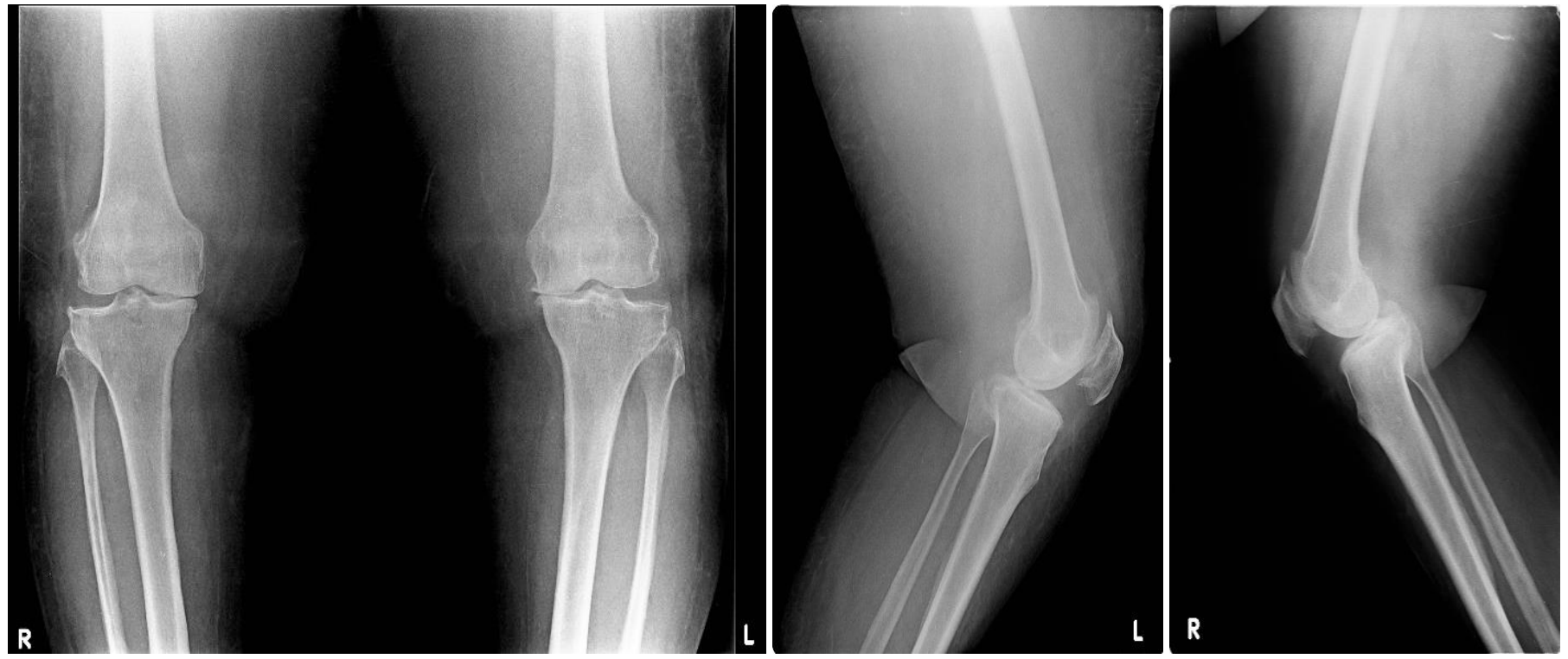

Pre-operative radiographs of osteoarthritis of both knees with varus deformity
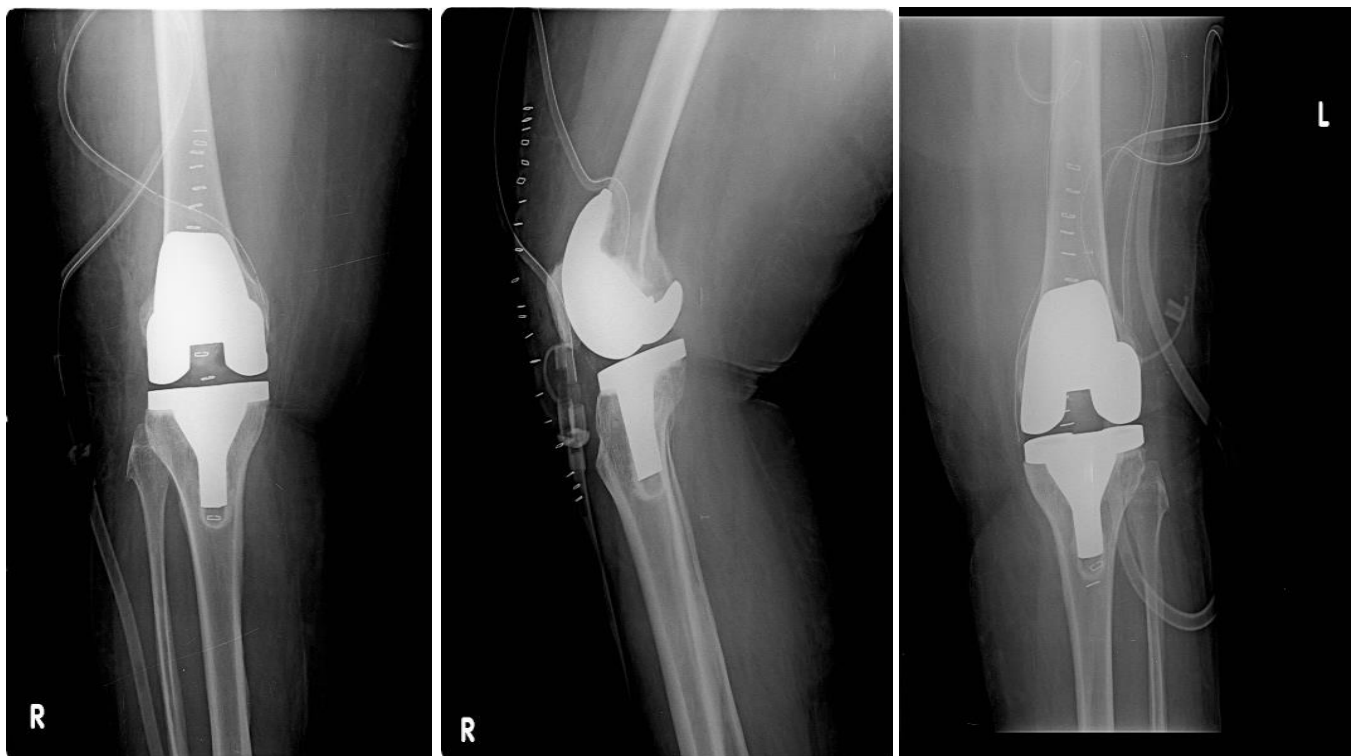

Post-operative radiographs of both knees

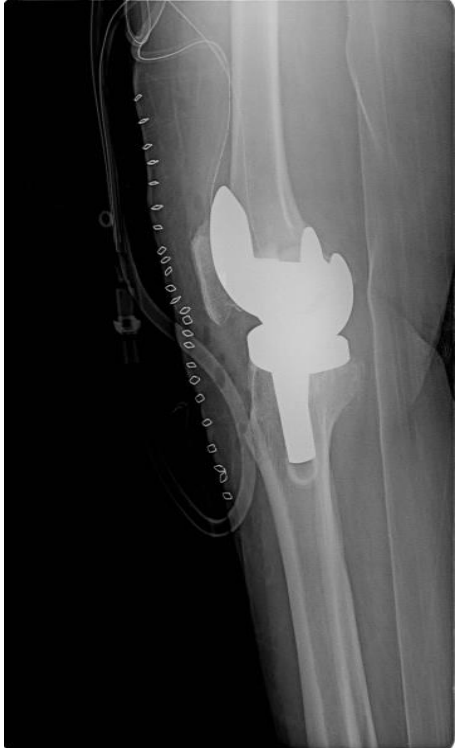




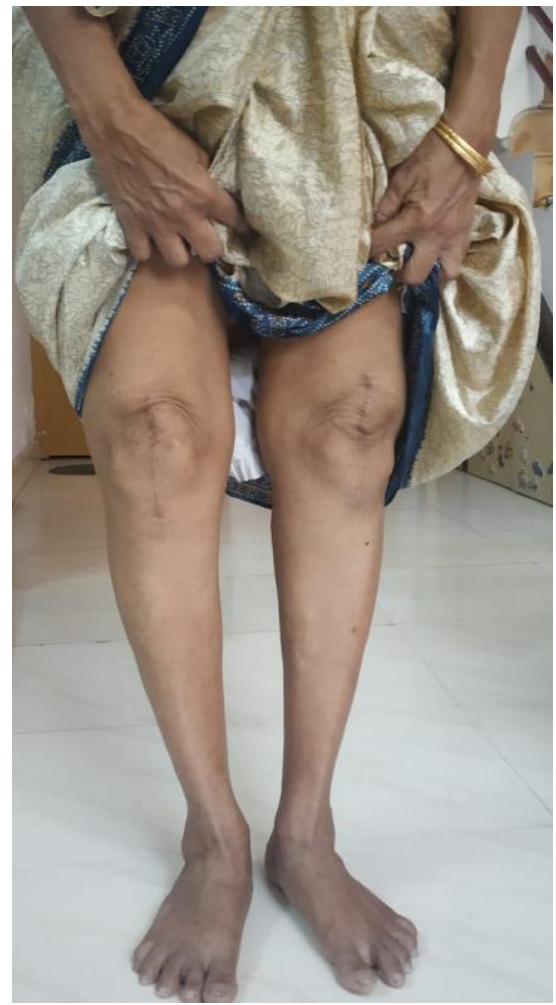

Bilateral TKA at four years follow-up.
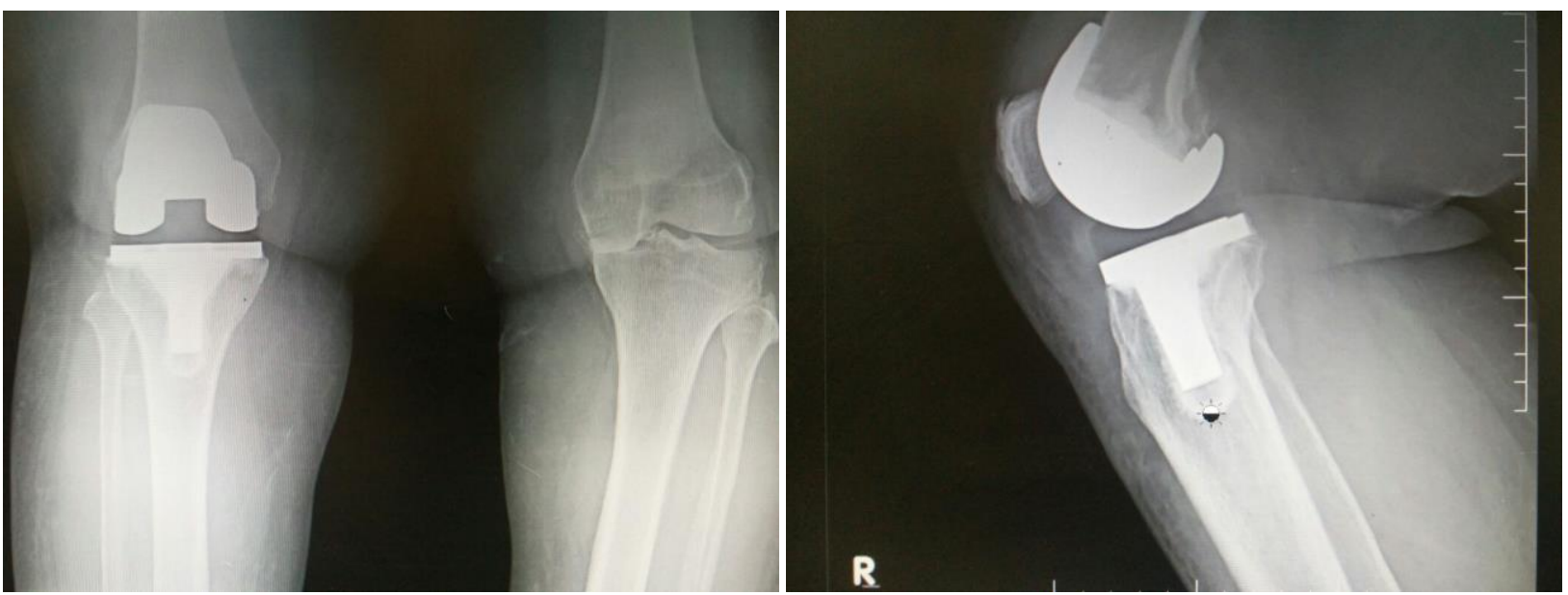

Unilateral TKA on right knee at three years follow-up, no evidence of lucencies

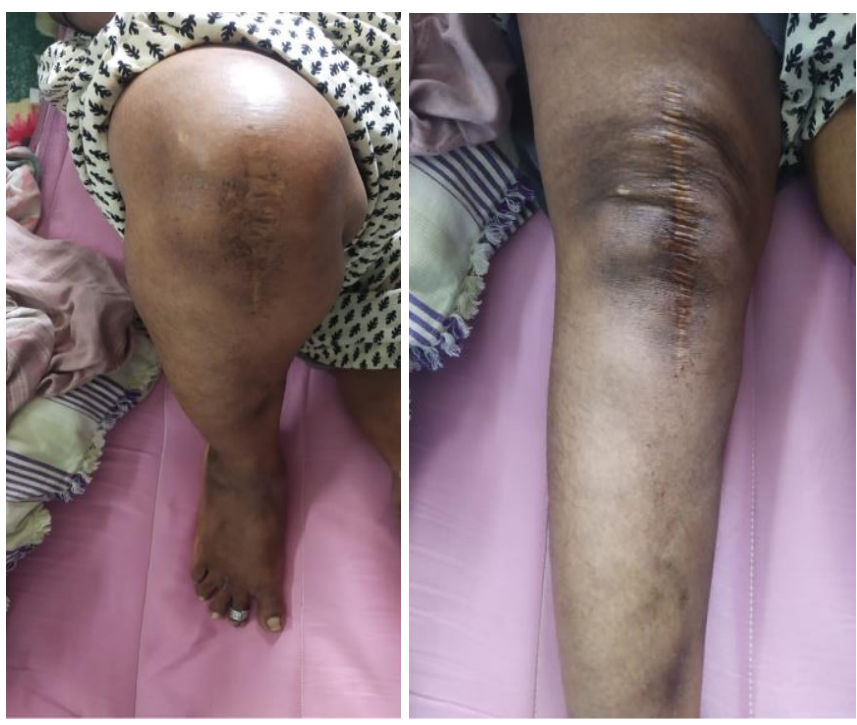

Clinical Photograph of same patient showing almost 120 degrees of knee flexion.

\section{Conclusion}

Total knee arthroplasty is an effective treatment for moderate to severe osteoarthritis of the knee in terms of improvement in health-related quality-of-life parameters. Preexisting comorbidities can influence the overall outcome following the procedure. A longer follow-up of a larger sample size will provide more insight into continued patient satisfaction for the expected life of the prosthesis.

\section{Reference}

1. Scott WN. Insall \& Scott Surgery of the Knee E-Book. Elsevier Health Sciences, 2011.

2. Liow Y, Wang W, Loh VW. Outpatient management of knee osteoarthritis. Singapore medical journal. 2017; 58(10):580.

3. Moskowitz RW, editor. Osteoarthritis: diagnosis and medical/surgical management. Lippincott Williams \& Wilkins, 2007.

4. Hochberg MC, Gravellese E, Silman A, Smolen J, Weinblatt M, Weisman M. Rheumatology. 7th ed. 
Philapelphia: Elsevier. 2018, 1560-1647.

5. Steinhaus ME, Christ AB, Cross MB. Total knee arthroplasty for knee osteoarthritis: support for a foregone conclusion? HSS Journal®. 2017; 13(2):207-10.

6. Dall'Oca C, Ricci M, Vecchini E, Giannini N, Lamberti $\mathrm{D}$, Tromponi C et al. Evolution of TKA design. Acta biomedica: Atenei Parmensis. 2017; 88(Suppl 2):17.

7. Insall JN, Dorr LD, Scott RD, Scott WN. Rationale of the Knee Society clinical rating system. Clin Orthop relat res. 1989; 248(248):13-4

8. APA Lombardi, Adolph V, Mallory, Thomas H, Fada, Robert A, Hartman et al. An Algorithm for the Posterior Cruciate Ligament in Total Knee Arthroplasty, Clinical Orthopaedics and Related Research ${ }^{\circledR}$ : 2001; 392:75-87

9. APA Scott W, Norman, Rubinstein, Michael. Posterior Stabilized Knee Arthroplasty: Six Years' Experience, Clinical Orthopaedics and Related Research: 1986; 205:138-145

10. Straw R, Kulkarni S, Attfield S, Wilton TJ. Posterior cruciate ligament at total knee replacement: Essential, Beneficial Or A Hindrance? The Journal of bone and joint surgery. British. 2003; 85(5):671-4.

11. Sehat KR, Evans R, Newman JH. How much blood is really lost in total knee arthroplasty?: correct blood loss management should take hidden loss into account. The Knee. 2000; 7(3):151-5.

12. Gao FQ, Li ZJ, Zhang K, Sun W, Zhang H. Four methods for calculating blood-loss after total knee arthroplasty. Chinese medical journal. 2015; 128(21):2856.

13. Good L, Peterson E, Lisander B. Tranexamic acid decreases external blood loss but not hidden blood loss in total knee replacement. British journal of anaesthesia. 2003 May 1; 90(5):596-9.

14. Wong J, Abrishami A, El Beheiry H, Mahomed NN, Davey JR, Gandhi R et al. Topical application of tranexamic acid reduces postoperative blood loss in total knee arthroplasty: a randomized, controlled trial. JBJS. 2010; 92(15):2503-13.

15. Yang ZG, Chen WP, Wu LD. Effectiveness and safety of tranexamic acid in reducing blood loss in total knee arthroplasty: a meta-analysis. JBJS. 2012; 94(13):1153-9.

16. Patel JN, Spanyer JM, Smith LS, Huang J, Yakkanti MR, Malkani AL. Comparison of intravenous versus topical tranexamic acid in total knee arthroplasty: a prospective randomized study. The Journal of arthroplasty. 2014; 29(8):1528-31.

17. An VV, Phan K, Levy YD, Bruce WJ. Aspirin as thromboprophylaxis in hip and knee arthroplasty: a systematic review and meta-analysis. The Journal of arthroplasty. 2016; 31(11):2608-16.

18. Meding JB, Meding LK, Ritter MA, Keating EM. Pain relief and functional improvement remain 20 years after knee arthroplasty. Clinical Orthopaedics and Related Research®. 2012; 470(1):144-9.

19. Barlow BT, Oi KK, Lee YY, Joseph AD, Alexiades MM. Incidence, indications, outcomes, and survivorship of stems in primary total knee arthroplasty. Knee Surgery, Sports Traumatology, Arthroscopy. 2017; 25(11):3611-9.

20. Rand JA, Bryan RS, Morrey BF, Westholm F. Management of infected total knee arthroplasty. Clinical orthopaedics and related research. 1986; (205):75-85.

21. Osmon DR, Berbari EF, Berendt AR, Lew D, Zimmerli W, Steckelberg JM et al. Diagnosis and management of prosthetic joint infection: clinical practice guidelines by the Infectious Diseases Society of America. Clinical infectious diseases. 2013; 56(1):e1-25. 